\title{
An Analytical Solution to the Stance Dynamics of Passive Spring-Loaded Inverted Pendulum with Damping
}

\author{
M. M. ANKARALI \\ Dept. of Electrical and Electronics Eng., \\ Middle East Tech. University, Ankara 06531, Turkey \\ E-mail: ankarali@eee.metu.edu.tr \\ O. ARSLAN* and U. SARANLI ${ }^{\dagger}$ \\ * Dept. of Electrical and Electronics Eng., \\ $\dagger^{\dagger}$ Dept of Computer Engineering, \\ Bilkent University, Bilkent Ankara 06800, Turkey \\ E-mail:*omur@ee.bilkent.edu.tr, †saranli@cs.bilkent.edu.tr
}

\begin{abstract}
The Spring-Loaded Inverted Pendulum (SLIP) model has been established both as a very accurate descriptive tool as well as a good basis for the design and control of running robots. In particular, approximate analytic solutions to the otherwise nonintegrable dynamics of this model provide principled ways in which gait controllers can be built, yielding invaluable insight into their stability properties. However, most existing work on the SLIP model completely disregards the effects of damping, which often cannot be neglected for physical robot platforms. In this paper, we introduce a new approximate analytical solution to the dynamics of this system that also takes into account viscous damping in the leg. We compare both the predictive performance of our approximation as well as the tracking performance of an associated deadbeat gait controller to similar existing methods in the literature and show that it significantly outperforms them in the presence of damping in the leg.
\end{abstract}

Keywords: SLIP, damping, legged locomotion, running robots, deadbeat control, analytical return maps

\section{Introduction}

Legged robot morphologies offer clear advantages on rough, outdoor terrain both in terms of the robustness and the diversity of their feasible locomotory behaviors. However, in light of complexities and inefficiency associated with slow, purely kinematic morphologies, it is fairly clear that legged systems can simultaneously achieve speed, agility and efficiency only by adopting dynamic modes of operation such as running and leaping. In this context, 
the Spring-Loaded Inverted Pendulum (SLIP) model is widely accepted in the literature as a very successful descriptive dynamical model for such behaviors. ${ }^{1-3}$ Not surprisingly, the same model has also been used as the basis of numerous robots capable of dynamic locomotion. ${ }^{4-8}$

Nevertheless, despite the apparent simplicity of this model, its dynamics during stance are nonintegrable, motivating a number of analytical approximations to support the analysis of its behaviors and the design of associated controllers. ${ }^{3,8-11}$ In this paper, we introduce a new analytical approximation method which takes into account damping in the leg, a dominant element for any physical legged robot often completely ignored by existing methods. We show that when the effects of damping are not neglected, our method significantly outperforms existing approximations in the literature ${ }^{3,10,12}$ both in its prediction of the stance map as well as its performance as a feed forward model within locomotion controllers.

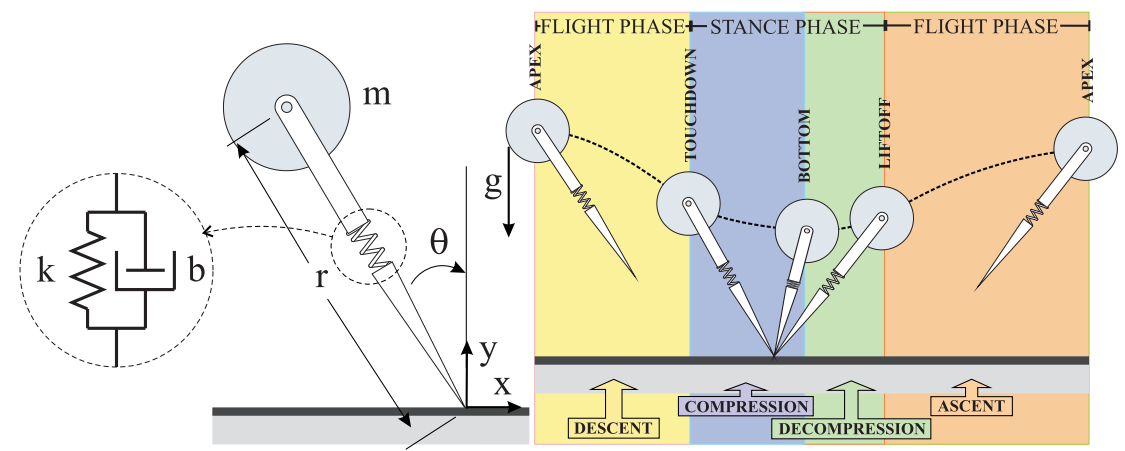

(a)

(b)

Fig. 1. The SLIP Model. (a) Coordinates and model parameters. (b) Locomotion phases (shaded regions) and transition events (boundaries).

\section{The SLIP Model and Dynamics}

Fig. 1 illustrates SLIP model we use, consisting of a point mass attached to a compliant, massless leg with viscous damping. Throughout locomotion, the model alternates as usual between stance and flight phases, which are further divided into compression, decompression and ascent, descent subphases respectively. Four important events define transitions between these subphases: touchdown, as the leg comes into contact with the ground, bottom, as the leg reaches its maximum compression, liftoff, as the toe takes off from the ground and finally apex, as the body reaches its maximum height during flight. Table 1 details the notation used throughout the paper. 
Table 1. Notation used throughout the paper

\begin{tabular}{|c|l|}
\hline \multicolumn{2}{|c|}{ SLIP States } \\
\hline$r, \theta, \dot{r}, \dot{\theta}$ & Leg length, leg angle and their derivatives \\
$p_{\theta}$ & Angular momentum during stance \\
$r_{e}, \theta_{e}, t_{e}$ & Leg length, leg angle and time at events $e \in\{t d, b, l o\}$, \\
& touchdown, bottom and liftoff, respectively. \\
$x, y, \dot{x}, \dot{y}$ & Horizontal and vertical body positions and velocities \\
$y_{a}, \dot{x} a$ & Body height and velocity at apex \\
\hline \multicolumn{2}{|c|}{ SLIP Parameters } \\
\hline$m, g$ & Body mass and gravitational acceleration \\
$l_{0}, k, b$ & Leg rest length and leg stiffness and damping coefficient \\
\hline
\end{tabular}

During flight, the body follows a ballistic trajectory, whereas during stance, the toe remains stationary on the ground with no torque applied to the leg. The stance dynamics of this model in polar leg coordinates are

$$
\begin{aligned}
m \ddot{r} & =m r \dot{\theta}^{2}+k\left(l_{0}-r\right)-m g \cos (\theta)-b \dot{r}, \\
0 & =\frac{d}{d t}\left(m r^{2} \dot{\theta}\right)+m g r \sin \theta .
\end{aligned}
$$

Subsequent sections present our analytical approximations to these dynamics as well as an associated deadbeat controller for SLIP running.

\section{An Approximate Stance Map for SLIP with Damping}

In deriving our analytical approximation to the stance dynamics of the SLIP model, we make the commonly used assumption that the leg remains close to the vertical throughout the entire stance phase. Consequently, the effects of gravity can be linearized around $\theta=0$. The resulting conservation of the angular momentum $p_{\theta}:=m r^{2} \dot{\theta}$ reduces the radial dynamics to

$$
\ddot{r}+b \dot{r} / m+k r / m-p_{\theta}^{2} /\left(m^{2} r^{3}\right)=-g+k l_{0} / m .
$$

Unfortunately, even these reduced dynamics do not admit an analytical solution. However, inspired by the method proposed by Geyer, ${ }^{10}$ we further assume that the relative spring compression, defined as $\frac{l_{0}-r}{l_{0}} \ll 1$, remains sufficiently small that the term $1 / r^{3}$ can be approximated by a Taylor series expansion around $l_{0}$, resulting in

$$
1 /\left.r^{3}\right|_{r=l_{0}} \approx 1 / l_{0}^{3}-3 / l_{0}^{4}\left(r-l_{0}\right)+O\left(\left(r-l_{0}\right)^{2}\right) .
$$

Under this approximation, (3) is reduced to

$$
\ddot{r}+(b / m) \dot{r}+\left(\omega_{0}^{2}+3 \omega^{2}\right) r=-g+l_{0} \omega_{0}^{2}+4 l_{0} \omega^{2},
$$

where we define $\omega:=\left(p_{\theta}\right) /\left(m l_{0}^{2}\right)$ and $\omega_{0}:=\sqrt{k / m}$. In order to solve (5) in a more compact form, we define $\hat{\omega}_{0}:=\sqrt{\omega_{0}^{2}+3 \omega^{2}}, F:=-g+l_{0} \omega_{0}^{2}+4 l_{0} \omega^{2}$, 
$\zeta:=b /\left(2 m \hat{\omega}_{0}\right)$ and $\omega_{d}:=\hat{\omega}_{0} \sqrt{1-\zeta^{2}}$. Assuming $\zeta<1$, we then have

$$
r=e^{-\zeta \hat{\omega}_{0} t}\left(A \cos \left(\omega_{d} t\right)+B \sin \left(\omega_{d} t\right)\right)+F / \hat{\omega}_{0}^{2},
$$

with $A$ and $B$ determined by touchdown states, $r_{t d}$ and $\dot{r}_{t d}$, as

$$
\begin{aligned}
& A=r_{t d}-F / \hat{\omega}_{0}^{2}, \\
& B=\left(\dot{r}_{t d}+\zeta \hat{\omega}_{0} A\right) / \omega_{d} .
\end{aligned}
$$

Simple differentiation yields the radial velocity as

$$
\dot{r}=-M e^{-\zeta \hat{\omega}_{0} t}\left(\zeta \hat{\omega}_{0} \cos \left(\omega_{d} t+\phi\right)+\omega_{d} \sin \left(\omega_{d} t+\phi\right)\right),
$$

where $M:=\sqrt{A^{2}+B^{2}}$ and $\phi:=\arctan (-B / A)$. Defining a new phase value, $\phi_{2}:=\arctan \left(-\sqrt{1-\zeta^{2}} / \zeta\right)$, the simplest form of the radial motion can be obtained as

$$
\begin{aligned}
& r(t)=M e^{-\zeta \hat{\omega}_{0} t} \cos \left(\omega_{d} t+\phi\right)+F / \hat{\omega}_{0}^{2}, \\
& \dot{r}(t)=-M \hat{\omega}_{0} e^{-\zeta \hat{\omega}_{0} t} \cos \left(\omega_{d} t+\phi+\phi_{2}\right) .
\end{aligned}
$$

Now that an analytical approximation to the radial trajectory is available, the angular trajectory can be determined using the constant angular momentum $\dot{\theta}=p_{\theta} /\left(m r^{2}\right)$. To this end, we linearize the term $1 / r^{2}$ around the rest length of the leg spring as

$$
1 /\left.r^{2}\right|_{r=l_{0}}=1 / l_{0}^{2}+2 / l_{0}^{3}\left(r-l_{0}\right)+O\left(\left(r-l_{0}\right)^{2}\right),
$$

and obtain an analytical solution for the rate of change of the leg angle as

$$
\dot{\theta}(t)=3 \omega-2 \omega F /\left(l_{0} \hat{\omega}_{0}^{2}\right)-2 \omega M e^{-\zeta \hat{\omega}_{0} t} \cos \left(\omega_{d} t+\phi\right) / l_{0},
$$

whose integral can then be used to determine the angular trajectory

$$
\theta(t)=\theta_{t d}+X t+Y\left(e^{-\zeta \hat{\omega}_{0} t} \cos \left(\omega_{d} t+\phi+\phi_{3}\right)-\cos \left(\phi+\phi_{3}\right)\right),
$$

where $X:=3 \omega-\frac{2 \omega F}{l_{0} \hat{\omega}_{0}^{2}}, Y:=\frac{2 \omega M}{l_{0} \hat{\omega}_{0}}$ and $\phi_{3}:=\arctan \left(\sqrt{1-\zeta^{2}} / \zeta\right)$. These approximations yield a sufficiently simple analytic solution to the stance dynamics of the SLIP model with damping, leaving only the determination of times at which the critical bottom and liftoff events occur during stance.

\section{Times for Critical Events: Bottom and Liftoff}

The bottom event is defined as the time at which the leg reaches its maximum compression with $\dot{r}\left(t_{b}\right)=0$. Using (9), this yields the solution

$$
t_{b}=\left(\pi / 2-\phi-\phi_{2}\right) / \omega_{d} .
$$

In contrast, the liftoff event occurs when the toe loses contact with the ground. Normally, when no damping is present in the leg with $\zeta=0,(6)$ 
becomes $r=M \cos \left(\hat{\omega}_{0}+\phi\right)+F / \hat{\omega}_{0}^{2}$, and an analytical solution for the liftoff time can easily be obtained. However, when damping is present in the system, the calculation of the liftoff time is considerably more difficult. This is because, regardless of the leg length, the SLIP toe leaves the ground when the ground reaction force induced by the leg crosses zero and starts to become negative. As a result, the liftoff time is computed as

$$
k\left(l_{0}-r\left(t_{l o}^{c 1}\right)\right)-b \dot{r}\left(t_{l o}^{c 1}\right)=0 .
$$

On the other hand, there are also cases where liftoff may be caused by explicit constraints imposed by the morphology of particular robots such as the BowLeg hopper. ${ }^{8}$ In this paper, we also allow explicit choice of the liftoff length, $r_{l o}$ by a high-level gait controller. In this case, the liftoff time is determined by the solution to the equation

$$
r\left(t_{l o}^{c 2}\right)=r_{l o} .
$$

The actual liftoff time can then be found as $t_{l o}=\min \left(t_{l o}^{c 1}, t_{l o}^{c 2}\right)$. Unfortunately, exact analytical solution of these equations is not possible and numerical methods, which are feasible due to the simple, one dimensional nature of these equations, need to be adopted.

Nevertheless, in order to preserve the fully analytic nature of the SLIP return map, we use a sufficiently accurate analytical approximation to compute both liftoff times. Since the exponential term multiplying the radial solution of (8) is the main source of the problem, we approximate it with its value at a specific instant during decompression as $e^{-\zeta \hat{\omega}_{0} t} \approx e^{-\zeta \hat{\omega}_{0} \gamma t_{b}}$, where $\gamma \geq 1$ is a tunable parameter. A reasonable choice is $\gamma=1+\left(r_{l o}-r_{b}\right) /\left(l_{0}-r_{b}\right)$, which incorporates the relative ratios of touchdown and liftoff lengths to estimate the liftoff time. Under this assumption, we have

$$
\begin{aligned}
& t_{l o}^{c 1} \approx\left(2 \pi-\arccos \left(k\left(l_{0}-F / \hat{\omega}_{0}^{2}\right) /\left(\bar{M} M e^{-\zeta \hat{\omega}_{0} \gamma t_{b}}\right)\right)-\phi-\phi_{4}\right) / \omega_{d} \\
& t_{l o}^{c 2} \approx\left(2 \pi-\arccos \left(\left(l_{0}-F / \hat{\omega}_{0}^{2}\right) /\left(M e^{-\zeta \hat{\omega}_{0} \gamma t_{b}}\right)\right)-\phi\right) / \omega_{d}
\end{aligned}
$$

where $\bar{M}:=\sqrt{\left(b \hat{\omega}_{0}\right)^{2}+k^{2}-2 k b \hat{\omega}_{0} \cos \left(\phi_{2}\right)} \& \phi_{4}:=\arctan \left(\frac{b \hat{\omega}_{0} \sin \left(\phi_{2}\right)}{b \hat{\omega}_{0} \cos \left(\phi_{2}\right)-k}\right)$. Finally, we perform a small correction on the liftoff angular velocity to account for the energy difference erroneously indiced by our approximations. While it is also possible to use gravity corrections on the angular momentum, ${ }^{11}$ the effect of this linearization is minimal compared to damping losses and this simple correction proved to be more than adequate. 


\section{Simulation Results}

\subsection{Predictive Performance}

In order to assess the performance of our method, we simulated a single stride of the SLIP model using a range of different initial conditions and damping coefficients, and compared its predictions to Geyer's ${ }^{10}$ analytic approximations. All simulations were done with $m=1$ and $r_{t d}=r_{l 0}=l_{0}=1$, together with initial conditions and remaining parameters accordingly scaled to be representative of natural runners, yielding 94248 simulations covering $\dot{x} \in[1,5](\mathrm{m} / \mathrm{s}), y \in[1.15,1.75](\mathrm{m}), k \in[250,2000](\mathrm{N} / \mathrm{m}), \theta_{t d_{\text {rel }}} \in$ $[-0.150 .25](\mathrm{rad}$.$) and \zeta_{0}:=b /(2 \sqrt{m k}) \in[0,0.5]$, where $\theta_{t d_{\text {rel }}}$ denotes the deviation of the touchdown angle from its value that would result in a neutral stride. For each simulation, we evaluated the performance of each approximation method using the percentage error $P E=100 \frac{\left\|x_{\text {true }}-x_{\text {approx }}\right\|_{2}}{\left\|x_{\text {true }}\right\|_{2}}$ associated with each relevant variable.

Table 2. Average percentage prediction errors for both Geyer's and our methods in predicting various elements of the SLIP state.

\begin{tabular}{|rl||c|c||c|c|}
\hline \multicolumn{1}{|c|}{} & \multicolumn{2}{|c||}{ Geyer's Approximation } & \multicolumn{2}{c|}{ Proposed Method } \\
\cline { 4 - 7 } & & $\mu \pm \sigma$ & $\max$ & $\mu \pm \sigma$ & $\max$ \\
\hline \hline liftoff pos. & $p_{l o}$ & $3.25 \pm 3.00$ & 21.37 & $0.36 \pm 0.44$ & 4.58 \\
\hline total energy & $E_{T}$ & $22.90 \pm 20.49$ & 102.95 & $0.05 \pm 0.08$ & 1.44 \\
\hline apex height & $h_{a}$ & $29.79 \pm 28.05$ & 170.07 & $0.11 \pm 0.36$ & 16.36 \\
\hline apex pos. & $p_{a}$ & $37.92 \pm 34.23$ & 170.78 & $0.28 \pm 0.84$ & 29.26 \\
\hline liftoff vel. & $v_{l o}$ & $45.52 \pm 46.74$ & 291.20 & $0.66 \pm 1.98$ & 61.23 \\
\hline stance time & $t_{s}$ & $9.28 \pm 8.35$ & 41.50 & $0.20 \pm 0.28$ & 3.23 \\
\hline
\end{tabular}
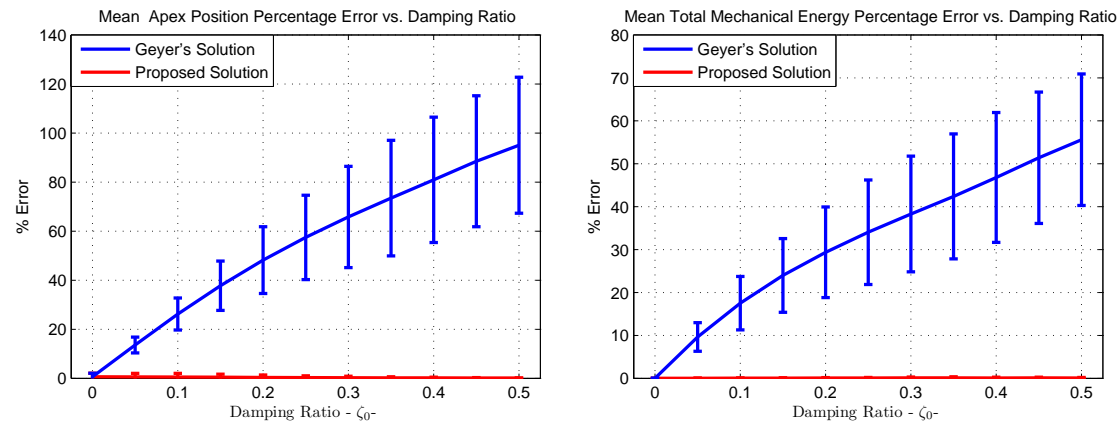

Fig. 2. Left: Average apex position prediction performance as a function of damping. Right: Average total mechanical energy prediction performance as a function of damping. The vertical bars represent the corresponding standard deviation. 
As shown in Table 2, the average predictive performance of our algorithm across the entire range of simulations is significantly better than that of Geyer's method..$^{10}$ Similarly, Fig. 2 illustrates the consistency of our approximations as a function of damping. Interestingly, our approximations perform better on the average with increased damping, which is due to the decreased amount of time spent in the stance phase when the radial velocity is decreased with higher damping in the leg.

\subsection{Tracking Performance under Gait Control}
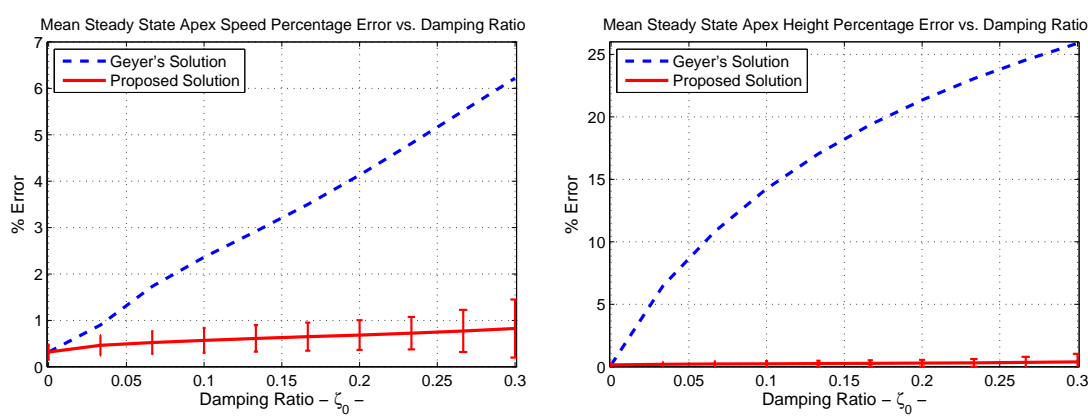

Fig. 3. Comparison of apex forward speed (left) and height (right) mean tracking errors at steady-state for a spring-mass runner with different damping coefficients in the leg.

In order to characterize the utility of our approximation method for the design of locomotion controllers, we compared the tracking performance of a deadbeat gait controller ${ }^{9}$ based on Geyer's approximations and our new method. Simulations were done with $m=1$ and $l_{0}=1$, covering $\dot{x}_{a} \in[1,4]$, $\dot{x}_{a}^{*} \in[1,4], y_{a} \in[1.5,1.8], y_{a}^{*} \in[1.5,1.8], k \in[1000,2000]$ and $\zeta_{0} \in[0,0.3]$, where $\dot{x}_{a}^{*}$ and $y_{a}^{*}$ denote the desired goal state.

Fig. 3 shows average steady state tracking errors for gait controllers based on Geyer's approximations and our method in trying to stabilize locomotion around the desired apex speed and height. Our results show that in both apex states of the SLIP, the tracking performance of the controller based on our algorithm outperforms existing alternatives in the presence of damping. Even though the accuracy" of both controllers decreases with increased damping, Geyer's map is much more sensitive to this parameter. The real difference between the controllers is seen in the apex height performance, which indicates the dominant effect of damping in the vertical dynamics. Overall, these results show that our analytic approximation provides a very accurate characterization of the SLIP stance dynamics for 
physical robot platforms where the effects of damping cannot be ignored.

\section{Conclusion}

In this paper, we proposed an analytical approximation to the stance dynamics of the Spring-Loaded Inverted Pendulum model that also takes into account non-negligible damping in the leg. Our simulation studies showed that both the predictive performance of our fully analytic approximations as well as the tracking performance of the resulting deadbeat controller significantly outperform existing approximation methods. We believe that such an accurate analytical stance map to the dynamics of the SLIP model will be invaluable in the design and analysis of physically realizable and effective controllers for dynamic legged locomotion.

\section{References}

1. R. M. Alexander, Three uses for springs in legged locomotion., International Journal of Robotics Research 9, 53 (1990).

2. R. Blickhan and R. J. Full, Similarity in multilegged locomotion: Bouncing like a monopode, J. of Comp. Physiology A: Neuroethology, Sensory, Neural, and Behavioral Phys. 173, 509(November 1993).

3. W. J. Schwind, Spring loaded inverted pendulum running: a plant model, PhD thesis, University of Michigan, (Ann Arbor, MI, USA, 1998).

4. M. H. Raibert, Legged robots that balance (MIT Pr., Cambridge, MA, 1986).

5. P. Gregorio, M. Ahmadi and M. Buehler, Design, Control, and Energetics of an Electrically Actuated Legged Robot, Transactions on Systems, Man, and Cybernetics 27, 626(August 1997).

6. S. G. Carver, Control of a spring-mass hopper, PhD thesis, Cornell University, (Ithaca, NY, USA, 2003).

7. J. W. Hurst, J. Chestnutt and A. Rizzi, Design and philosophy of the BiMASC, a highly dynamic biped, in Proc. of the Int. Conf. On Robotics and Automation, (Pittsburgh, PA, 2007).

8. G. Zeglin, The Bow Leg Hopping Robot, PhD thesis, Carnegie Mellon University, (Pittsburgh, PA, USA, 1999).

9. U. Saranli, Dynamic locomotion with a hexapod robot, $\mathrm{PhD}$ thesis, University of Michigan, (Ann Arbor, MI, USA, 2002).

10. H. Geyer, A. Seyfarth and R. Blickhan, Spring-mass running: simple approximate solution and application to gait stability, Journal of Theoretical Biology 232, 315(Feb 2005).

11. O. Arslan, U. Saranlı and O. Morgül, An aproximate stance map of the spring mass hopper with gravity correction for nonsymmetric locomotions, in Proc. of the Int. Conf. on Robotics and Automation, (Kobe, Japan, 2009).

12. W. J. Schwind and D. E. Koditschek, Approximating the Stance Map of a 2 DOF Monoped Runner, Journal of Nonlinear Science 10, 533 (2000). 